\title{
Development of a clinical score to distinguish malignant from benign esophageal disease in an undiagnosed patient population referred to an esophageal diagnostic assessment program
}

\author{
Negar Ahmadi ${ }^{1}$, Lawrence Mbuagbaw ${ }^{2,3,4}$, Waël C. Hanna ${ }^{1}$, Christian Finley ${ }^{1}$, John Agzarian ${ }^{1}$, \\ Chuck K. Wen ${ }^{5}$, Michal Coret ${ }^{1}$, Colin Schieman ${ }^{6}$, Yaron Shargall ${ }^{1}$ \\ ${ }^{1}$ Division of Thoracic Surgery, McMaster University/St. Joseph's Healthcare Hamilton, Hamilton, ON, Canada; ${ }^{2}$ Department of Health Research \\ Methods, Evidence and Impact, McMaster University, Hamilton, ON, Canada; ${ }^{3}$ Biostatistics Unit, Father Sean O'Sullivan Research Centre, St \\ Joseph's Healthcare Hamilton, Hamilton, ON, Canada; ${ }^{4}$ Centre for the Development of Best Practices in Health, Yaoundé, Cameroon; ${ }^{5}$ Division of \\ Thoracic Surgery, University of British Columbia, Surrey, BC, Canada; ${ }^{6}$ Section of Thoracic Surgery, University of Calgary, Calgary, AB, Canada \\ Contributions: (I) Conception and design: Y Shargall, N Ahmadi, L Mbuagbaw; (II) Administrative support: Y Shargall, M Coret; (III) Provision of \\ study materials or patients: WC Hanna, C Finley, J Agzarian, CK Wen, C Schieman, Y Shargall; (IV) Collection and assembly of data: M Coret; (V) \\ Data analysis and interpretation: L Mbuagbaw; (VI) Manuscript writing: All authors; (VII) Final approval of manuscript: All authors. \\ Correspondence to: Yaron Shargall, MD, FRCSC. McMaster University/St. Joseph's Healthcare Hamilton, 50 Charlton Avenue East T-2105, \\ Hamilton, ON L8N 4A6, Canada. Email: shargal@mcmaster.ca.
}

Background: Esophageal cancer is associated with poor prognosis. Diagnosis is often delayed, resulting in presentation with advanced disease. We developed a clinical score to predict the risk of a malignant diagnosis in symptomatic patients prior to any diagnostic tests.

Methods: We analyzed data from patients referred to a regional esophageal diagnostic assessment program between May 2013 and August 2016. Logistic regression was performed to identify predictors of malignancy based on patient characteristics and symptoms. Predicted probabilities were used to develop a score from 0 to 10 which was weighted according to beta coefficients for predictors in the model. Score accuracy was evaluated using a receiver operating characteristic (ROC) curve and internally validated using bootstrapping techniques. Patients were classified into low (0-2 points), medium (3-6 points), and high (7-10 points) risk groups based on their scores. Pathologic tissue diagnosis was used to assess the effectiveness of the developed score in predicting the risk of malignancy in each group.

Results: Of 530 patients, 363 (68\%) were diagnosed with malignancy. Factors predictive of malignancy included male sex, family history of cancer and esophageal cancer, fatigue, chest/throat/back pain, melena and weight loss. These factors were allocated 1-2 points each for a total of 10 points. Low-risk patients had $70 \%$ lower chance of malignancy $(\mathrm{RR}=0.28,95 \% \mathrm{CI}: 0.21-0.38)$, medium-risk had $50 \%$ higher chance of malignancy ( $\mathrm{RR}=1.5,95 \% \mathrm{CI}: 1.26-1.77)$, and high-risk patients were 8 times more likely to be diagnosed with malignancy $(\mathrm{RR}=8.2,95 \% \mathrm{CI}: 2.60-25.86)$. The area under the ROC curve for malignancy was 0.82 (95\% CI: 0.77-0.87).

Conclusions: A simple score using patient characteristics and symptoms reliably distinguished malignant from benign diagnoses in a population of patients with upper gastrointestinal symptoms. This score might be useful in expediting investigations, referrals and eventual diagnosis of malignancy.

Keywords: Esophageal cancer; diagnostic assessment program; esophageal cancer screening; dysphagia

Submitted Nov 18, 2019. Accepted for publication Dec 23, 2019.

doi: $10.21037 /$ jtd.2020.02.07

View this article at: http://dx.doi.org/10.21037/jtd.2020.02.07

(c) Journal of Thoracic Disease. All rights reserved. 


\section{Introduction}

Esophageal cancer is the eight most common cancer diagnosed worldwide with some geographical variations in histology and epidemiology (1). In North America and Europe, squamous cell carcinoma (SCC) of the esophagus has been decreasing while esophageal adenocarcinoma (EAC) rates are on the rise $(2,3)$. In Canada, rates of EAC have doubled in the last 20 years. This rising rate of EAC in Canada is associated with poor survival, with reported 5 -year survival rate of about $13 \%$ in Canada (4). The poor prognosis associated with esophageal cancer is in part due to the late presentation of the disease, with at least half the patients being diagnosed with stage IV (5). Although population screening for esophageal cancer has been advocated in some Asian countries with higher incidence of esophageal cancer $(6,7)$, there exists no North American or European guideline on screening for esophageal cancer (8). Studies assessing the cost-effectiveness of esophageal cancer screening in North America have yielded mixed results with most studies failing to show improvements in long-term survival (9-11). Patients with advance esophageal cancer may present with some upper gastrointestinal symptoms. About half the patients diagnosed with esophageal cancer have a history of heartburn or acid regurgitation $(12,13)$. Dysphagia has been reported as a common presenting symptom in esophageal cancer and is often associated with fatigue and weight loss (14). While dysphagia is an alarming symptom, it has been reported to be relatively prevalent in the general non-cancer population, with an incidence of up to $20 \%$ in the elderly (15). Incidence of malignancy in patients presenting with dysphagia is estimated to be about 10-15\% (16-19). Symptoms other than dysphagia and their predictive ability to diagnose esophageal cancer have not yet been well studied.

The Esophageal Diagnostic Assessment Program (EDAP) at our institution is a recently developed program composed of various disciplines aiming at rapid assessment of patients referred for symptoms of dysphagia along with other symptoms potentially indicative of upper gastroesophageal malignancies. However, as such symptoms can be non-specific and in a significant portion of patients become associated with benign diagnosis, developing a tool to predict the risk of malignancy based on a number of risk factors can help allocate limited resources more efficiently. The aim of this study was to design and validate a simple clinical scoring system to identify high risk patients referred to our EDAP with upper gastrointestinal symptoms prior to performing any diagnostic tests.

\section{Methods}

This is a retrospective analysis of prospectively collected data from patients referred to a regional EDAP at St Joseph's Healthcare Hamilton, McMaster University between May 2013 and August 2016. Patients can be referred to our EDAP if they are found to have any concerning symptoms that might suggest an underlying upper gastrointestinal malignancy requiring further investigations. In addition, patients may also be referred by endoscopists if they have suspected or proven diagnosis of esophageal cancer. All referred patients were contacted prior to their first clinical appointment by a medical clerk unaware of the diagnosis. A comprehensive battery of questions was then completed collecting demographic data, medical history and clinical symptoms, without a knowledge of patient's diagnosis (Tables 1 and 2). The information was recorded prospectively in a computerized database. All patients underwent an esophagogastroduodenoscopy (EGD) as the initial diagnostic assessment. The diagnosis of esophageal cancer was made based on pathological confirmation from endoscopic biopsy samples demonstrating invasive carcinoma. Those diagnosed with esophageal cancer completed further staging investigations. A 10-point questionnaire was then developed retrospectively from the initial battery of questions used prospectively at the initial telephone intake and applied to the entire study cohort, assigning each patient as either low, medium or high risk based on cut-off probabilities. A comparison was then made between the questionnaire prediction and the actual diagnosis.

Approval for this study was obtained from our institutional Research Ethics Board (Hamilton Integrated Research Ethics Board, REB\#13-218). Informed consent from the participants was not required for this study.

\section{Statistical analysis}

Statistical comparisons of the two groups (benign and malignant) were performed using the univariate Chisquare test for categorical variables and Student's t-test for continuous variables. Binary logistic regression was used to identify independent predictors of malignancy. Variables included in the model were age (years), sex (male, female), alcohol use (none, socially, weekly, daily, more than 1 per day, heavy), family history of cancer (yes/no), smoking history (never, former, current) and symptoms of dysphagia, loss of appetite, fatigue, hoarse voice, regurgitation, indigestion, nausea, heartburn, cough, 
Table 1 Baseline characteristics of the patients in benign and malignant groups

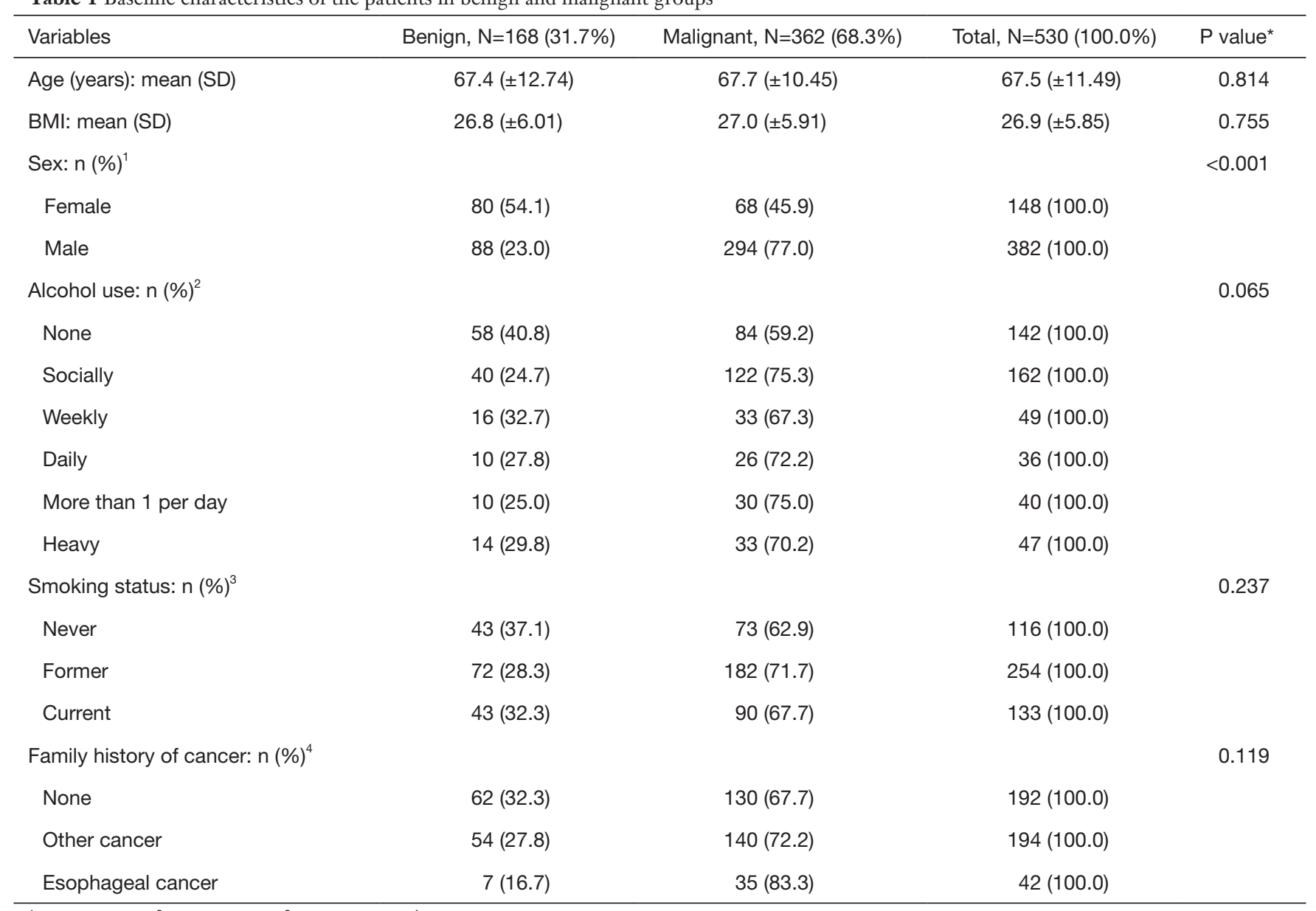

${ }^{1}, 39$ missing; ${ }^{2}, 93$ missing; ${ }^{3}, 66$ missing; ${ }^{4}, 141$ missing; *, Chi-squared or $t$-tests.

melena, odynophagia, chest throat or back pain, vomiting, hemoptysis and weight loss (all categorized as yes/no). A backwards elimination method (threshold of $\mathrm{P}<0.5$, and relative contribution to the model) was used to determine the final variables for the predictive model with the outcome of diagnosis of malignancy. Hosmer and Lemeshow's test was used to assess goodness of fit (20). Predictive models may have an overestimated performance when determined on the sample of subjects used to build the model. Therefore, internal validation is required to provide more reliable estimates of performance. Bootstrapping is an internal validation approach that provides stable estimates with low bias (21). The bootstrap technique draws random samples from the original data with replacement and model performance is estimated for each sample. This process generates a sampling distribution of the statistic of interest, which are used to provide more accurate estimates. We ran
100 bootstrap samples $(\mathrm{N}=530)$, using the simple sampling approach (case resampling with replacement from the original dataset), with $95 \%$ confidence intervals estimated using the percentile approach. The predicted probabilities (the probability of each patient being diagnosed with malignancy) were used to develop a score out of 10 , with higher score more likely to result in a malignant diagnosis. The individual score for each variable included in the final model was weighted according to the relative contributions of that variable using the beta coefficients. Accordingly, each variable was allocated a maximum score of 1 or 2 points. For clinical application the total score was divided into three categories (likelihood of esophageal cancer): low [0-2], medium [3-6] and high [7-10]. The area under the receiver operating characteristics (ROC) curve was then calculated. Statistical analysis was performed using SPSS $^{\oplus}$ version 14 (SPSS, Chicago, Illinois, USA). 
Table 2 Distribution of symptoms experienced by participants in the benign and malignant groups

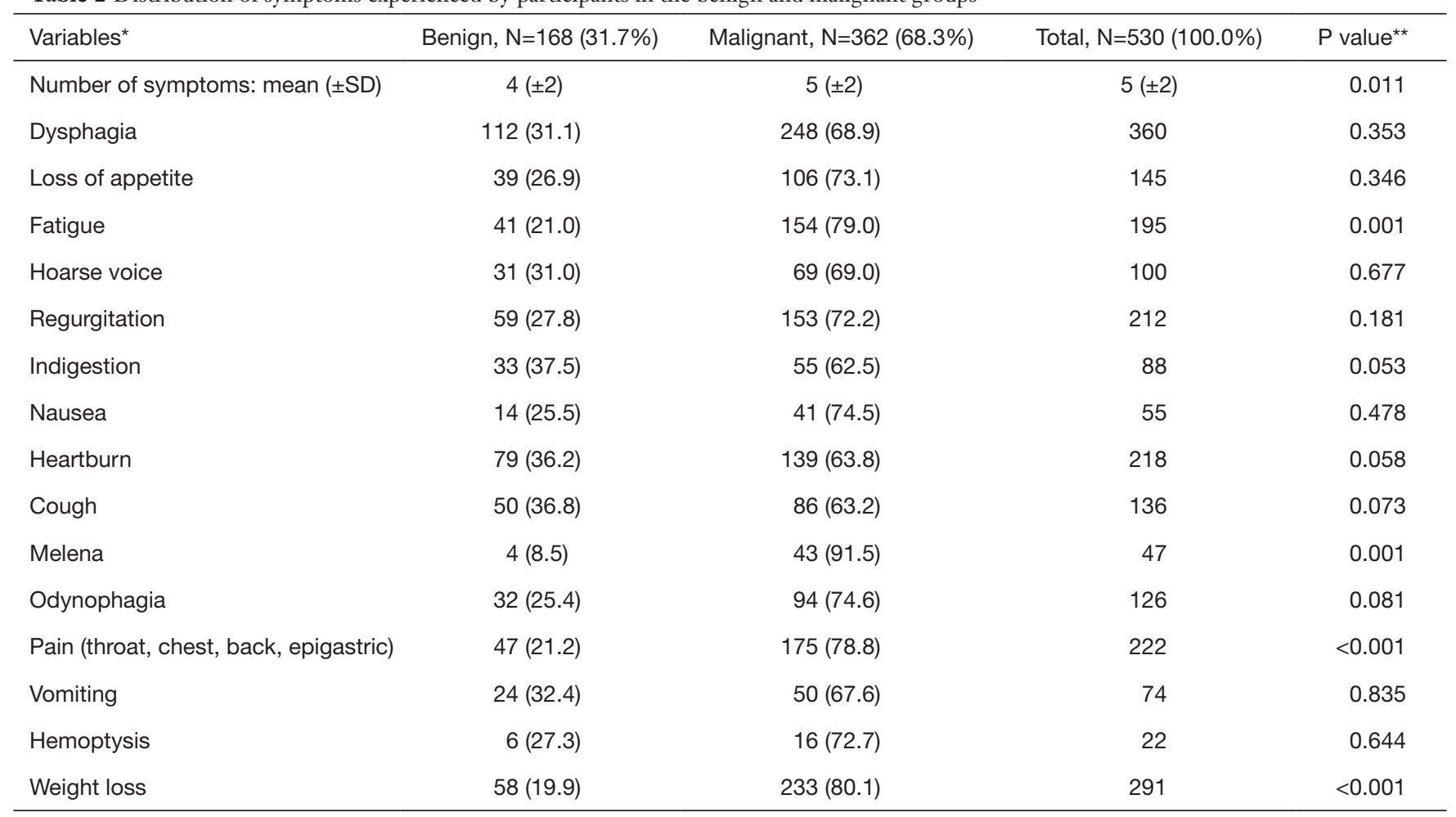

${ }^{*}$, missing data: $10-34 \% ;{ }^{* *}$, Chi-squared or $t$-test.

\section{Results}

From May 2013 to August 2016, 530 patients were referred to the EDAP at our institution. Of those, 368 $(62 \%)$ were eventually diagnosed with esophageal or gastroesophageal junction cancer. There were more males in the malignant group, while age, body mass index (BMI), alcohol consumption, smoking history and a history of other cancers or family history of cancer did not differ between the groups. Table 1 summarizes the baseline characteristics of the patients included in the study based on their final diagnosis. Of all malignant and dysplasia diagnoses, 287 (79\%) were adenocarcinoma, 58 (16\%) were SCC and 17 (5\%) were Barrett's esophagus. Amongst those with benign diagnoses, the prevalence of inflammatory esophageal disease was 27 (16\%), hiatal hernia was $23(14 \%)$, benign stricture was $11(7 \%)$ and motility disorder was $10(6 \%)$. Seventy five patients of the benign group (45\%) did not have a specific etiology of their symptoms identified based on their endoscopic assessment. Table 2 summarizes the distribution of the presenting symptoms based on the final diagnoses. Dysphagia was the most common presenting symptom in the entire population $(n=360,68 \%)$, followed by weight loss $(n=291,55 \%)$. While prevalence of dysphagia was not found to significantly differ between the malignant and benign groups $(\mathrm{P}=0.35)$, fatigue, weight-loss, melena and throat/neck/chest pains were associated with an eventual diagnosis of malignancy in the univariate analysis.

After backwards elimination, the final variables retained in the model to develop the scoring system were sex, family history of cancer, family history of esophageal cancer, fatigue, pain in chest, throat or back, age, melena and weight loss. The strongest predictor of esophageal cancer was male sex (OR 6.13; 95\% CI: 3.33-11.24), followed by family history of esophageal cancer (OR 4.07; $95 \%$ CI: 1.21-13.68) and melena (OR 3.39; 95\% CI: 1.05-10.9). Table 3 outlines the final model.

The scoring system (out of 10) devised from the model based on the log odds of having esophageal cancer allocated two points each for male sex, family history of esophageal cancer and melena and one point each for family history of cancer, fatigue, chest/throat/ epigastric/back pain and weigh loss as shown in Figure 1. Patients were classified into three risk groups based on their score (low: 0-2, medium: 3-6, high: 7-10). Model fit was 
Table 3 Final predictive model for malignant diagnosis

\begin{tabular}{|c|c|c|c|c|c|c|c|}
\hline Variables & $\beta$ & OR & \multicolumn{3}{|c|}{$95 \% \mathrm{Cl}$ for OR } & \multicolumn{2}{|c|}{ Bootstrap } \\
\hline Sex (male) & 1.813 & 6.126 & 3.339 & 11.238 & 0.000 & 0.010 & 0.115 \\
\hline Family history of cancer (yes) & 0.783 & 2.188 & 1.210 & 3.958 & 0.010 & 0.010 & -0.027 \\
\hline Family history of esophageal cancer (yes) & 1.403 & 4.068 & 1.209 & 13.683 & 0.023 & 0.020 & 0.338 \\
\hline Pain in throat, chest or back (yes) & 0.991 & 2.695 & 1.488 & 4.881 & 0.001 & 0.010 & 0.339 \\
\hline Age (years) & 0.026 & 1.026 & 1.001 & 1.052 & 0.040 & 0.040 & 0.002 \\
\hline Melena (yes) & 1.220 & 3.387 & 1.053 & 10.895 & 0.041 & 0.010 & 0.683 \\
\hline Weight loss (yes) & 0.929 & 2.532 & 1.438 & 4.458 & 0.001 & 0.010 & 0.060 \\
\hline
\end{tabular}

*, the difference between the mean of the bootstrap beta-coefficient and the regression beta coefficient. Smaller values are better.

\begin{tabular}{|c|c|c|}
\hline \multicolumn{2}{|l|}{ Risk factors } & Score \\
\hline \multirow{2}{*}{ Sex } & Male & 2 \\
\hline & Female & 0 \\
\hline \multirow{2}{*}{$\begin{array}{l}\text { Family history of } \\
\text { esophageal cancer }\end{array}$} & Yes & 2 \\
\hline & No & 0 \\
\hline \multirow{2}{*}{ Melena } & Yes & 2 \\
\hline & No & 0 \\
\hline \multirow{2}{*}{ Family history of cancer } & Yes & 1 \\
\hline & No & 0 \\
\hline \multirow{2}{*}{ Fatigue } & Yes & 1 \\
\hline & No & 0 \\
\hline \multirow{2}{*}{$\begin{array}{l}\text { Pain (chest/throat/ } \\
\text { epigastric/back) }\end{array}$} & Yes & 1 \\
\hline & No & 0 \\
\hline \multirow{2}{*}{ Weight loss } & Yes & 1 \\
\hline & No & 0 \\
\hline \multicolumn{2}{|l|}{ Total score (/10) } & \\
\hline
\end{tabular}

Figure 1 Esophageal cancer screening questionnaire. Low risk: 1-2, medium risk: 3-6, high risk: 7-10.

very good (Hosmer and Lemeshow goodness of fit test: Chisquare $=5.8 ; \mathrm{df}=8 ; \mathrm{P}=0.670$ ). After bootstrapping, bootstrap estimates and significance tests for the Beta coefficient were similar and bias was low (as shown in Table 3).

The score in its current form has area under the ROC curve of 0.82 (95\% CI: 0.77-0.87; P<0.001) as shown in Figure 2.

Table 4 summarized the distribution of benign and malignant disease in the different risk groups (low, medium and high). In this sample, patients classified as low risk (0-2 points) had a $70 \%$ lower chance of malignancy (RR $=0.28 ; 95 \%$ CI: $0.21-0.38 ; \mathrm{P}<0.001)$. Patients classified

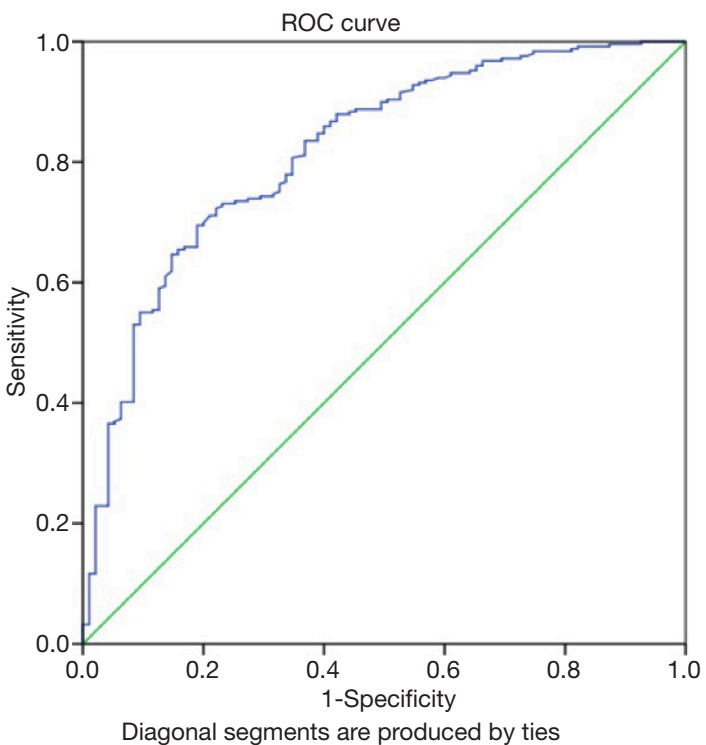

Figure 2 Area under the ROC curve for malignancy prediction score. ROC, receiver operating characteristic.

as medium risk ( 3 to 6 points) had a $50 \%$ higher chance of malignancy ( $R R=1.5 ; 95 \% C I: 1.26-1.77 ; \mathrm{P}<0.001)$. Patients classifies as high risk ( 7 to 10 points) were 8 times more likely to be diagnosed with malignancy $(\mathrm{RR}=8.2$; 95\% CI: 2.60-25.86; $\mathrm{P}<0.001)$.

\section{Discussion}

The rising rates of EAC in North America and the lack of a cost-effective screening tool has contributed to the high rates of advanced-stage diagnoses and poor survival of esophageal 
Table 4 Prevalence of benign and malignant disease in the three predicted risk groups

\begin{tabular}{lccc}
\hline \multirow{2}{*}{ Outcome } & \multicolumn{3}{c}{ Prediction score } \\
\cline { 2 - 4 } & Low risk [0-2] & Medium risk [3-6] & High risk [7-10] \\
\hline Benign & $85(62 \%)$ & $80(24 \%)$ & $3(5 \%)$ \\
Malignant & $52(38 \%)$ & $257(76 \%)$ & $53(95 \%)$ \\
Total & $137(100 \%)$ & $337(100 \%)$ & $56(100 \%)$ \\
\hline
\end{tabular}

cancer $(14,22)$. EGD remains the gold standard for diagnosis of esophageal cancer. Given the current incidence of esophageal cancer in North America, mass population screening has not been recommended by any North American or European societies $(23,24)$. The quick and simple questionnaire proposed here to identify those symptomatic patients at high risk of malignancy classifies patients into three risk categories. Patients identified as high risk (score 7-10) were found to be 8 times more likely to have diagnoses of esophageal malignancy and therefore might benefit from an expedited care. This questionnaire is most applicable to a tertiary care centre responsible for care of patients with esophageal disease and can be administered by a healthcare professional responsible for the intake of consults in an attempt to expedite the initial clinic visits and further investigations for patients identified as high risk of esophageal malignancy.

Esophageal cancer is often asymptomatic until it progresses to advance disease. On the other hand, nonspecific upper gastroenterology symptoms are often not investigated due to their high prevalence in older population which ultimately leads to delays in diagnosis. In order to improve early diagnosis of esophageal cancer, studies have assessed the association between esophageal cancer and various upper gastrointestinal symptoms such as dysphagia. A number of previous studies have attempted to identify patients at high risk of malignancy amongst those who present with dysphagia. The Edinburg Dysphagia Score (EDS) is an example of a scoring system devised to identify high risk patients referred for dysphagia and includes age, sex, weight loss, duration of symptoms, localization of dysphagia and acid reflux as risk factors for malignancy (25). Another similar study showed malignancy to be more common in older men with a shorter duration of symptoms (less than 8 weeks) and associated weight loss but no associated reflux symptoms (18). While dysphagia remains a common presentation in patients with esophageal cancer, its high prevalence in various benign conditions takes away from its diagnostic predictability of esophageal cancer (16-19).
In our study, although the prevalence of dysphagia was higher in the malignant group (69\% vs. 31\%), this difference did not reach significance. The difference between our findings and previous studies that have reported a significant association between dysphagia and esophageal cancer might lie in the inherent nature of our referrals not being primarily for symptoms of dysphagia, whereas previous studies have mainly assessed the risk of esophageal cancer in patients presenting predominantly with symptoms of dysphagia $(18,25,26)$. Our study is unique in this regard by having a wider referral pattern than primarily dysphagia.

Amongst all other presenting symptoms assessed in our study, melena was the strongest predictor of esophageal cancer (OR: 3.4) followed by sensation of pain in throat, back, epigastric or chest (OR: 2.7) as well as weight loss (OR: 2.5) and fatigue (OR: 2.4). Weight loss has been shown to be a significant predictor of esophageal malignancy by other studies $(18,25,26)$. The association between esophageal cancer and pain (abdominal and epigastric pain) has also been shown in a British database review (27). Very few studies have reported on the association between esophageal cancer and the remaining symptoms mentioned above. Our study showed that male sex, family history of esophageal cancer and family history of other malignancies are all significant risk factors of esophageal malignancy. The association between family history and esophageal cancer has been studied previously (28-30). Studies have shown family history to be a risk factor for SCC, however the evidence for the association between family history and EAC is somewhat mixed. The results of our study are novel in showing an association between esophageal cancer and family history of not only esophageal cancer but also other malignancies.

In our study, although rates of smokers and heavy alcohol drinkers were higher in the esophageal malignancy group compared to the benign group, they were not independent predictors of malignancy. Smoking and alcohol drinking and their association with esophageal cancer in patients presenting with dysphagia has not been well examined in studies conducted in Europe including the study that reported on the EDS by Rhatigan et al. $(18,25)$. A Japanese study by Tsukamoto and colleagues (26) reported smoking and heavy alcohol drinking to be significantly associated with esophageal malignancy. The observed difference between our findings and those of Tsukamoto $e t a l$. and other studies from Asia might be due to the difference in geographical variations of histology of esophageal cancer with SCC being more prevalent in Asia compared to North America. Previous studies have included age as a predictor of malignancy (25). In our study, age was a significant predictor, however, it 
contributed very little in the final model and therefore was not included. In this study, BMI was also not included in the final model as there was no significant difference in the BMI across the two groups. However, this could be partly due to the missing BMI value for portion of the patients.

Our study has some limitations. The study population included all patients referred to a regional tertiary care centre for management of esophageal diseases and includes those with symptoms concerning for upper gastrointestinal malignancy as well as those with confirmed pathological diagnoses of esophageal cancer by a prior endoscopic assessment. Therefore, the study population is pre-selected to have more prevalence of malignancy compared to other studies that included patients mainly based on their symptom presentation. This is further demonstrated in the high prevalence of esophageal cancer diagnosis in the study population (68\%). This population is representative of most referral patterns to centres that treat esophageal cancer in Canada and the United States. However, the nature of the study population limits the generalizability of the results to the primary care population, although it does give a useful starting point for initial assessment and decision making in patients with worrisome symptoms.

The retrospective nature of the study resulted in some missing data as shown in Table 1 which might affect the association between some of the risk factors such as alcohol consumption or BMI and malignant diagnosis. Another limitation of this study compared to prior studies in this area is the lack of information on the quantity of weight loss as well as the duration of symptoms. While some studies have shown certain quantity of weight loss (often $>3 \mathrm{~kg}$ ) and short duration of symptoms ( $<8$ weeks) to be associated with diagnosis of esophageal malignancy $(18,25)$, we were not able to specifically assess those associations as this information was not collected as part of the data collection. Another limitation of this study is that the assessment tool proposed here has not yet been validated in another population. Although bootstrapping methods did show satisfactory internal validity, external validity of this questionnaire in another population remains an important next step. Finally, although the tool can help us prioritize patients at high risk of esophageal cancer, those identified as low risk still require relatively urgent attention as $38 \%$ were still found to have a malignant diagnosis. In spite of those limitations, we believe that this score will be useful in triaging and prioritizing further investigations for all patients with upper GI symptoms and will serve as a tool for clinicians who often encounter those symptoms in their patients.
In summary, in patients referred for suspected or diagnosed esophageal cancer, male patients with family history of esophageal cancer or other malignancies as well as symptoms of melena, weight loss, fatigue and pain (throat, chest or back) are more likely to have an eventual diagnosis of esophageal cancer. We have developed a simple assessment tool that can be applied prior to the first clinic visit to all patients referred to an EDAP to identify those at high risk of esophageal malignancy and potentially expedite their assessment and care accordingly. We recommend further testing of this tool in routine clinical practice.

\section{Acknowledgments}

Funding: None

\section{Footnote}

Conflicts of Interest: YS serves as the unpaid editorial board member of Fournal of Thoracic Disease from Aug 2019 to Jul 2021. The other authors have no conflicts of interest to declare.

Ethical Statement: The authors are accountable for all aspects of the work in ensuring that questions related to the accuracy or integrity of any part of the work are appropriately investigated and resolved. Approval for this study was obtained from our institutional Research Ethics Board (Hamilton Integrated Research Ethics Board, REB\#13-218). Informed consent from the participants was not required for this study.

Open Access Statement: This is an Open Access article distributed in accordance with the Creative Commons Attribution-NonCommercial-NoDerivs 4.0 International License (CC BY-NC-ND 4.0), which permits the noncommercial replication and distribution of the article with the strict proviso that no changes or edits are made and the original work is properly cited (including links to both the formal publication through the relevant DOI and the license). See: https://creativecommons.org/licenses/by-nc-nd/4.0/.

\section{References}

1. Kamangar F, Dores GM, Anderson WF. Patterns of cancer incidence, mortality, and prevalence across five continents: defining priorities to reduce cancer disparities in different geographic regions of the world. J Clin Oncol 2006;24:2137-50. 
2. Torre LA, Siegel RL, Ward EM, et al. Global Cancer Incidence and Mortality Rates and Trends--An Update. Cancer Epidemiol Biomarkers Prev 2016;25:16-27.

3. Pohl H, Sirovich B, Welch HG. Esophageal adenocarcinoma incidence: are we reaching the peak? Cancer Epidemiol Biomarkers Prev 2010;19:1468-70.

4. Otterstatter MC, Brierley JD, De P, et al. Esophageal cancer in Canada: trends according to morphology and anatomical location. Can J Gastroenterol 2012;26:723-7.

5. Canadian Cancer Society's Steering Committee on Cancer Statistics. Canadian Cancer Statistics. In: Society CC. editor. Toronto 2010.

6. Gao QY, Fang JY. Early esophageal cancer screening in China. Best Pract Res Clin Gastroenterol 2015;29:885-93.

7. Chiu PWY, Uedo N, Singh R, et al. An Asian consensus on standards of diagnostic upper endoscopy for neoplasia. Gut 2019;68:186-97.

8. Zakko L, Lutzke L, Wang KK. Screening and Preventive Strategies in Esophagogastric Cancer. Surg Oncol Clin N Am 2017;26:163-78.

9. Corley DA, Levin TR, Habel LA, et al. Surveillance and survival in Barrett's adenocarcinomas: a population-based study. Gastroenterology 2002;122:633-40.

10. Gupta N, Bansal A, Wani SB, et al. Endoscopy for upper GI cancer screening in the general population: a costutility analysis effect of a prior endoscopy on outcomes of esophageal adenocarcinoma among United States veterans. Gastrointest Endosc 2011;74:610-24.e2.

11. Rubenstein JH, Sonnenberg A, Davis J, et al. Effect of a prior endoscopy on outcomes of esophageal adenocarcinoma among United States veterans. Gastrointest Endosc 2008;68:849-55.

12. Lagergren J, Bergstrom R, Lindgren A, et al. Symptomatic gastroesophageal reflux as a risk factor for esophageal adenocarcinoma Meta-analysis: the association of oesophageal adenocarcinoma with symptoms of gastrooesophageal reflux. N Engl J Med 1999;340:825-31.

13. Rubenstein JH, Taylor JB. Meta-analysis: the association of oesophageal adenocarcinoma with symptoms of gastrooesophageal reflux. Aliment Pharmacol Ther 2010;32:1222-7.

14. Mansour NM, Groth SS, Anandasabapathy S. Esophageal Adenocarcinoma: Screening, Surveillance, and Management. Annu Rev Med 2017;68:213-27.

15. Easterling CS, Robbins E. Dementia and dysphagia. Geriatr Nurs 2008;29:275-85.

16. Kapoor N, Bassi A, Sturgess R, et al. Predictive value of alarm features in a rapid access upper gastrointestinal cancer service. Gut 2005;54:40-5.

17. Melleney EM, Subhani JM, Willoughby CP, et al. Dysphagia referrals to a district general hospital gastroenterology unit: hard to swallow, Predictive value of symptoms and demographics in diagnosing malignancy or peptic stricture. Dysphagia 2004;19:78-82.

18. Murray IA, Palmer J, Waters C, et al. Predictive value of symptoms and demographics in diagnosing malignancy or peptic stricture. World J Gastroenterol 2012;18:4357-62.

19. Eslick GD, Talley NJ. Dysphagia: epidemiology, risk factors and impact on quality of life--a population-based study. Aliment Pharmacol Ther 2008;27:971-9.

20. Lemeshow S, Hosmer DW, Jr. A review of goodness of fit statistics for use in the development of logistic regression models. Am J Epidemiol 1982;115:92-106.

21. Steyerberg EW, Harrell FE Jr, Borsboom GJ, et al. Internal validation of predictive models: efficiency of some procedures for logistic regression analysis. J Clin Epidemiol 2001;54:774-81.

22. Ro TH, Mathew MA, Misra S. Value of screening endoscopy in evaluation of esophageal, gastric and colon cancers. World J Gastroenterol 2015;21:9693-706.

23. Shaheen NJ, Falk GW, Iyer PG, et al. ACG Clinical Guideline: Diagnosis and Management of Barrett's Esophagus. Am J Gastroenterol 2016;111:30-50; quiz 1.

24. Fitzgerald RC, di Pietro M, Ragunath K, et al. British Society of Gastroenterology guidelines on the diagnosis and management of Barrett's oesophagus. Gut 2014;63:7-42.

25. Rhatigan E, Tyrmpas I, Murray G, et al. Scoring system to identify patients at high risk of oesophageal cancer. Br J Surg 2010;97:1831-7.

26. Tsukamoto M, Manabe N, Kamada T, et al. Number of Gastrointestinal Symptoms is a Useful Means of Identifying Patients with Cancer for Dysphagia. Dysphagia 2016 31:547-54.

27. Stapley S, Peters TJ, Neal RD, et al. The risk of oesophago-gastric cancer in symptomatic patients in primary care: a large case-control study using electronic records. Br J Cancer 2013;108:25-31.

28. Chen T, Cheng H, Chen X, et al. Family history of esophageal cancer increases the risk of esophageal squamous cell carcinoma. Sci Rep 2015;5:16038.

29. Dhillon PK, Farrow DC, Vaughan TL, et al. Family history of cancer and risk of esophageal and gastric cancers in the United States. Int J Cancer 2001;93:148-52.

30. Jiang X, Tseng CC, Bernstein L, et al. Family history of cancer and gastroesophageal disorders and risk of esophageal and gastric adenocarcinomas: a case-control study. BMC Cancer 2014;14:60.

Cite this article as: Ahmadi N, Mbuagbaw L, Hanna WC, Finley C, Agzarian J, Wen CK, Coret M, Schieman C, Shargall Y. Development of a clinical score to distinguish malignant from benign esophageal disease in an undiagnosed patient population referred to an esophageal diagnostic assessment program. J Thorac Dis 2020;12(3):191-198. doi: 10.21037/jtd.2020.02.07 